\title{
Features of Laser Tube Bending processing based on Laser Forming: A Review
}

\author{
Khalil Ibrahe \\ Ahmad $^{4}$ \\ ${ }^{1}$ Department of Mechanical and Manufacturing Engineering, Faculty of Engineering, University Putra Malaysia, Malaysia \\ ${ }^{2}$ Laser Research Centre, Ministry of Science and Technology, Baghdad, Iraq \\ ${ }^{3}$ Faculty of Science, University Putra Malaysia, Malaysia \\ ${ }^{4}$ Faculity of Science, Al-Nahrain University, Baghdad, Iraq
}

\begin{abstract}
Laser forming of materials can help produce the desired shapes from sheets or tubes, which is not possible through conventional methods. Molds, dies, and external force are not needed for the laser forming of materials. Furthermore, the process is flexible and can be controlled by making changes to the materials, their geometry, and the laser parameters, either individually or in combination with each other. Investigations into laser tube bending are scarce in the literature pertaining to this field of study despite its potential and industrial importance. In contrast, much research has been conducted on laser sheet forming, which employs the same mechanism. In this study, laser tube bending is compared with laser sheet forming in order to develop a better understanding of the laser forming process. This review elucidates upon the mechanisms employed in laser forming (in general) and laser tube bending (in particular). A number of investigation methods are reviewed and analytical models of the process are also described. The principle of laser tube bending is explained, and the influence of various parameters (such as materials, geometry, laser, edge, and cooling effects) on the process is analyzed. Additionally, large tubes bending and preloading, or laser assistance, are examined.
\end{abstract}

Keywords: Material processing; Laser forming; Laser tube bending; Laser mechanism; Scanning speed

\section{Introduction}

Laser forming was first reported and subsequently used in the 1980s; after this, laser material processes became widespread in many applications [1]. Laser forming is recommended for accurate and controlled bending, as well as, for the prevention of defects that may occur during conventional forming methods; such as thinning, wrinkling, spring back and cross-section distortion [2]. Moreover, laser forming is a thermo-mechanical forming process that can be used to realize more complex shapes without the application of external forces or dies [1]. The specimen is irradiated by a laser beam to produce rapid localized heating and followed by free cooling once the laser is turned off or moved to next zone [3]. The heated area experiences wall thickening and compressive plastic deformation due to thermal expansion that is constrained by the unheated material; as a result, the irradiated material will undergo a shortening in the axial of the specimen's direction, in this case forcing it to bend towards the laser source [4]. Laser forming is a complex process that involves more than one nonlinear phenomenon [5]. Recently, there are many crucial applications that utilize laser forming in different fields, such as aerospace, automation, heat exchangers and shipbuilding [6]. There are three significant categories of parameters involved in laser forming. The first would be material specification parameters, such as coefficient of absorption, thermal expansion coefficient, the density and specific heat, as well as, the mechanical properties such as the yield stress, Young's modulus, and Poisson's ratio. The second is laser parameters such as wavelength, laser power, laser beam diameter and feed rate or scanning speed. The third include geometric parameters, such as sheet dimensions or tube diameter and thickness, depending on the specimen's shape [7]. In order to understand the behavior of laser forming, one must grasp the key elements that control the process trends and the metal deformation by analyzing the three main mechanisms of the laser forming process [8].

\section{Laser Forming Mechanisms}

\section{Temperature gradient mechanism (TGM)}

TGM is a common mechanism used to bend sheet metal towards a laser light source. The situations for the temperature gradient mechanism are energy parameters that lead to a steep temperature gradient across the sheet thickness [9]; as shown in Figure 1a Furthermore, TGM can be used to bend sheets and is typically realized when the laser beam diameter that incidents on the specimen surface are almost equal to or larger than the sheet thickness [10,11] (Figure 1).

The scanning speed should be relatively fast when the thermal conductivity of the material is high in order to maintain the temperature gradient during the bending process [12]. Moreover, different thermal expansions generate different thermal strains on the adjacent layers of the material. The elastic expansion changes to plastic deformation because the heated metal faces a restricting force from the unheated material [13]. Several researchers had focused their efforts on creating an analytical model to estimate the value of the bending angle. The conclusions were that the most crucial material parameters affecting the process are the thermal expansion coefficient and heat capacity [14].

*Corresponding author: Khalil Ibraheem Imhan, Department of Mechanical and Manufacturing Engineering, Faculty of Engineering, University Putra Malaysia, 43400 UPM Serdang-Selangor Darul Ehsan, Malaysia, Tel: +60 1-800-22-5587; E-mail: theeqar@live.com

Received January 06, 2018; Accepted January 25, 2018; Published February 01, 2018

Citation: Imhan KI, Baharudin BTHT, Zakaria A, Ismail MISB, Alsabti NMH, et al. (2018) Features of Laser Tube Bending processing based on Laser Forming: A Review. J Laser Opt Photonics 5: 174. doi: 10.4172/2469-410X.1000174

Copyright: @ 2018 Imhan $\mathrm{KI}$, et al. This is an open-access article distributed under the terms of the Creative Commons Attribution License, which permits unrestricted use, distribution, and reproduction in any medium, provided the original author and source are credited. 


\section{Buckling mechanism (BM)}

Generally, BM is used to bend thin sheets and tubes with a small temperature gradient through the material thickness, which is negligible [15]. Moreover, the laser beam diameter is much greater than the material's thickness, and in some cases, by a factor of ten. This is to allow more energy to penetrate the material [16]. Hence, the scanning speed should be slower than the TGM's case in order to achieve a uniform distribution of temperature in the direction of thickness. Due to thermal expansion, elastic buckling takes place, followed by plastic deformation which causes the formation of a convex [17]. BM, as seen in Figure $1 \mathrm{~b}$, is recommended for the production of complex shapes with three-dimensional profiles [18]. An analytical model was used to express the relationship between the bending angle and the main effective parameters in the bending process for thin sheets and tubes. This review focuses on tube bending, and the analytical model will be clarified in the following subsections.

\section{Upsetting mechanism (UM)}

Some researchers regard the UM as a part of BM because the conditions are similar, such as the laser beam diameter and scanning speed; however, the material geometry increases in thickness and its section modulus changes [19]. UM is applied when there is no temperature gradient via the material thickness, as shown in Figure 1c. The thermal expansion in the heated area is incapable of generating enough stress to bend the specimen; accordingly, the plastic compressive stress is restricted from the unheated area. The material cools after the laser spot moves, which shortens the specimen due to the increasing thickness of the heated area [20].

\section{Laser Forming Investigation Methods}

The methods employed to study the forming process can be classified into experimental and simulation via analytical or finite element analyses. However, the experimental results are true only for the investigated cases. The analytical case can be more specific, while the finite element simulation agrees with the experimental results [21].

\section{Experimental method}

Generally, the experimental method is the used in the initial attempts to examine any phenomena that accompany any new method or technique as a point of reference. Hennige et al. [22] conducted experimental investigations on laser bending using TGM by the $\mathrm{CO}_{2}$ laser system with the power of $815 \mathrm{~W}$ and path feed rate of $33.3 \mathrm{~m} / \mathrm{s}$ to irradiated mild steel (St14/SAE), the investigations shows that an improvement of working accuracy in laser bending can be achieved by a closed loop control of the process. Lawrence et al. [23] utilized a highpowered diode laser to bend mild steel and induced a bending angle, which increased with an increasing number of irradiations and high laser powers. Gollo et al. [24] applied the Nd-Yag laser with the power of $500 \mathrm{~W}$ to experimentally and numerically study the temperature distribution in a sheet of mild steel. The numerical and experimental results are relatively in good agreement. The increase in laser power led to an increase in bending angle. Liu et al. [25] elaborated upon the mechanism of the laser bending of tube parts and experimentally studied the process parameters. The results indicated that the bending angle increases when the laser power increases and the scanning speed decreases.

\section{Analytical method}

An analytical model is another method that can be used to understand the bending process and control the parameters to predict the results [26]. There are two aspects of the process that must be calculated: the temperature field, and the stresses due to the temperature field. The plastic flow during laser heating is a complex phenomenon, thus, the researchers focused on finding an analytical model to represent the TGM process. Within this direction and orientation, Shen and Vollertsen [27], in their review, referred to the expression that was derived based on a two-layer model for TGM to elucidate the best condition for line laser forming of sheet metals. It depends on material properties, specimen geometry and laser beam specifications, which can be represented as:

$$
\alpha_{b}=\left(3 \alpha_{t h} P A\right) /\left(\rho c_{p} v s^{2}\right)
$$

where $\alpha_{b}$ is the bending angle, $\alpha_{t h}$ is the coefficient of thermal expansion of the workpiece, $P$ is the laser power, $A$ is the absorption coefficient, $\rho$ is the density, $c_{p}$ is the specific heat capacity, $v$ is the velocity and $s$ are the sheet thickness. Yau et al. [28] added the counter-bending influence (which mean that the workpiece initially bends to opposite direction of laser source) in order to provide an explanation for some of the purely elastic straining. This modification resulted in two equations: one for the counter-bending angle which occurs at the heat cycle, and one for the bending angle at the end of the cooling cycle. The ultimate equation (a)

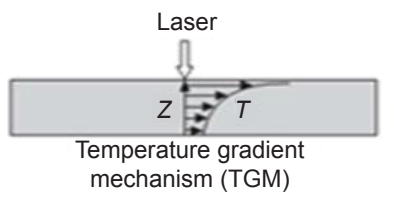

(b)

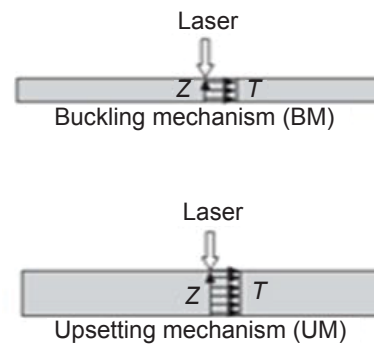

(c)
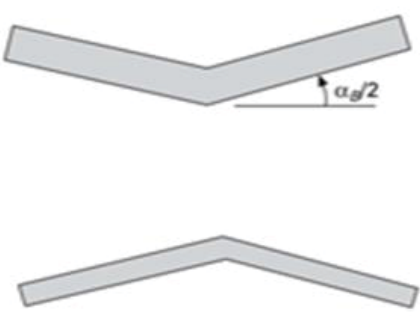

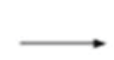

Figure 1: Types of laser forming mechanisms: (a) Temperature Gradient Mechanism (TGM), (b) Buckling Mechanism (BM) and (c) Upsetting Mechanism (UM) [11]. 
for the bending angle counting the temperature field equation in Yau's model is:

$$
\alpha_{b}=\left(\left(2 l \alpha_{t h} P A\right) /\left(2 \rho c_{p} v s^{2}\right)\right)-(36 l Y / s E)
$$

where $l$ is the heated zone half-length, $\mathrm{E}$ is Young's modulus and $\mathrm{Y}$ is the yield stress. Cheng and Lin [29] proposed and developed an analytical model to assess the angle formed by a laser, which is produced during the heating and cooling processes; thereby, reducing the average error for this model to $0.082^{\circ}$. Moreover, Cheng et al. [30] studied the experimental, analytical and numerical methods to realize the bending mechanism and the effect of parameters on the process specifications of varying thickness plates. Hence, the assessing the thermo-mechanical behavior for the processing, according to the heating of the material and uneven stresses and strains generated.

\section{Numerical method}

Currently, computer science is undergoing tremendous development in both information quality and processing speed. Laser forming is a complex thermo-mechanical process, and the specimen's temperature, specifications, and dimensions are a function of time and direction, depending on the variables. Hence, numerical analysis is suitable for modeling the process and explaining the behavior during forming with sufficient software packages, such as ANSYS and ABAQUS. Li and Yao [31] studied the strain rate effects, which are temperature dependent, and a "constant peak temperature" method was developed with the help of numerical modeling and solution. Moreover, Kyrsanidi et al. [32] numerically simulated the bending and formation of the sine-shaped plates. He proposed a new algorithm that can calculate the temperature, depending on time, stress and strain, as well as, predict the final form of steel plates. The numerical results were then validated by using the experimental results conducted by the $\mathrm{CO}_{2}$ laser with $3 \mathrm{~kW}$. In their study, Lok and Hideki [33] proposed a new method to calculate the $3 \mathrm{D}$ temperature distribution during laser forming by utilizing the Quasi-Monte Carlo integration. This improved the calculation speed to 1 hour instead of 36 hours in FEM. Shi et al. [8] reported a numerical study for the laser forming of plates by employing two laser beams scanning simultaneously along two parallel lines. They demonstrated that plastic deformation is larger compared to when using one scanning laser. Wang et al. [34] simulated the process of laser bending, and the FEM software ANSYS was applied to predict the stress-strain and heat temperature fields. Safari [35] numerically examined the effects of the laser and material, as well as, the geometrical parameters on the laser bending process by using the ABAQUS software. The results illustrated that the bending angle increases with increasing laser power and decreases with increasing scanning speed, sheet thickness, and beam diameter. Moslemi Naeini et al. [36] investigated the process of line-type heating by a laser using analytical and experimental methods. The analyses were performed in a commercial package of the ANSYS software. Coordinate Measurement Machine (CMM) was employed to measure the bending angle. The most affected factor on the bending angle is the thickness of the sheet metal. Chen and $\mathrm{Xu}$ [37] performed a fully 3D finite element analysis to simulate the thermo-elasto-plastic deformation process during laser forming. The computational and experimental results were in excellent agreement. Jamil et al. have presents experimental results and a 3D finite element model for simulation of laser micro-tubes bending process. The numerical model was used to study the effect of bending control parameters; laser power, pulse length and initial displacement at the tube's free end on output properties; temperature, bending angle, ovality, and bulging was successfully demonstrated by varying the. A commercial finite element analysis software package ANSYSs was used to develop the model. Temperature-dependent thermal and mechanical properties were considered in the analysis [38].

\section{Laser Tube Bending (LTB)}

Laser tube bending is a promising approach for producing specified shapes of curved tubes, which can be applied in numerous artificial applications; such as boilers, engines, heat exchangers and air conditioners. Moreover, it can be used to overcome the defects associated with conventional bending methods such as wrinkling, wall thinning, springbuck and cross-sectional distortion [2]. The geometry of the tube has made the laser forming process more complicated. Hence, research was conducted to understand and standardize the behavior of the process mechanisms.

\section{Principle of laser tube bending}

Laser tube bending is similar to the heat treatment process, where the surface temperature of the material must be less than the melting point. There are several nonlinear phenomena accompanying this process such as temperature, microstructure Figure 2 [39], and stress field changes; all of which are significantly correlated [1]. Hao and Li [40] proposed a new analytical model to determine the bending angle and study the effects of the significant parameters. Circular tube with circumferential scanning was used, as shown in Figure 2, where the tube rotates on its axial axis around $180^{\circ}$ and the laser light is defocused on the tube surface with the laser beam diameter being much larger than the tube's thickness. The heated area experiences wall thickening and compressive plastic deformation due to thermal expansion limitation by the unheated material. When the laser source is turned off, the material shrinks on its heated surface part. This results in the shortening of the irradiated material in the axial direction of the tube, which forces it to bend towards the laser source [41]. Guan et al. used the finite element simulation method process is combined with the genetic algorithms and laser bending of tubes process is enhanced based on different objection functions [42]. During the bending process, heat is generated due to strain energy, but it is very small compared to the input laser beam energy, which makes it negligible.

\section{Analytical modelling of laser tube bending}

There are two analytical models that control laser tube bending: the thermal model and the mechanical model that involves stress converted to plastic strain to produce a bending angle. Moreover, there are other aspects of the thermal model related to the temperature field caused by a laser beam, assuming uniform thermal conductivity and

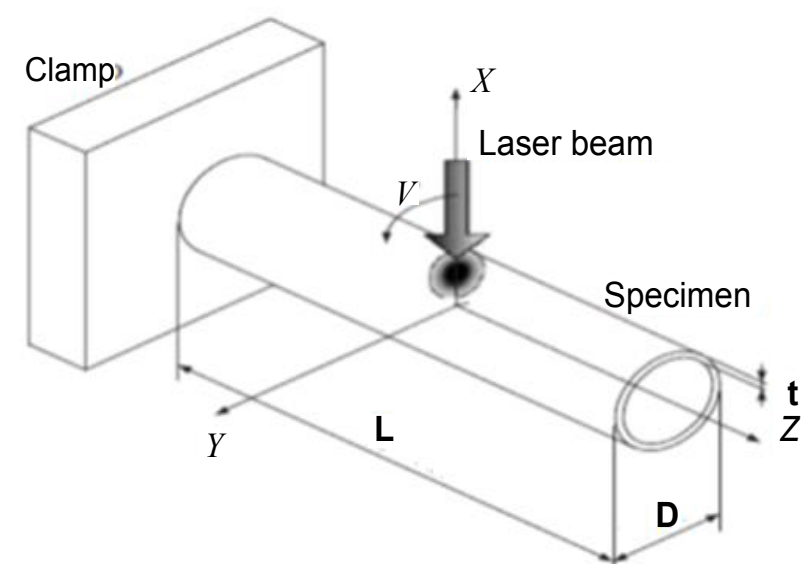

Figure 2: Laser tube bending circumferential scanning scheme. 
the temperature of the heat source acting in a quasi-steady method. Hence, the temperature can be determined from [41]:

$$
\left.T(z, t)=(2(1-R) F / k)(S t / \pi)^{1 / 2}\right) \operatorname{ierfc}\left(z / 2(k t)^{1 / 2}\right)
$$

where $k$ is the thermal conductivity, $F$ is the heat flux at the spot's center, $i e r f c$ is the integral complementary error function, $R$ is the reflectivity, $S$ is the material diffusivity, $z$ is the material thickness and $t$ is the heating time. To determine the temperature on the material surface when $z=0$, the eqn. (4) needs to be:

$$
T(0, t)=(2(1-R) F / k)(S t / \pi)^{1 / 2}
$$

where $k$ is the thermal conductivity coefficient and $S$ is the thermal diffusivity coefficient.

Laser intensity is $F=P / A$, where $P$ is the laser power and $A$ is the heating area, which can be written as:

$$
A=\pi D l / 2
$$

where $D$ is the tube diameter and $l$ is the laser beam diameter. The heat loss from the specimen per unit area $\mathrm{W} / \mathrm{m}^{2}$ is either the result of convection [43]:

$$
q_{c}=h_{c}\left(T_{s}-T_{a}\right)
$$

where $h_{c}$ is the coefficient of convection heat transfer, $T_{s}$ is the tube surface temperature and $T_{a}$ is air temperature.

Or radiation, which can be expressed as:

$$
q_{r}=5.67 \times 10^{8} \varepsilon\left(T_{s}^{4}-T_{a}^{4}\right)
$$

where $\varepsilon$ is the surface Emissivity [43].

The laser heats the material surface and induces plastic compressive strains. From Figure 3, there are two forces, F, exerted inversely from each other between the heated and unheated material, where laser scanning only takes place on the upper portion of the tube circumference and in the axial direction [40].

The strain on the scanning area is:

$$
\varepsilon_{s}=-\Delta T \alpha / 2+F / S E
$$

where $S$ is the area of the section of the half tube, and $E$ is Young's modulus of the material.

The strain of the un-scanned portion is:

$$
\varepsilon_{u s}=-F / S E
$$

The angle bend can be found from:

$$
\theta=(\pi l / 4 D)\left[\Delta T \alpha-2(\sqrt{ } 2-1) \sigma_{y} / E\right]
$$

where $l$ is the length of the heated region, and the laser beam diameter, $D$, can be considered as the tube diameter, while $\sigma_{y}$ is the yield stress of the material [40].
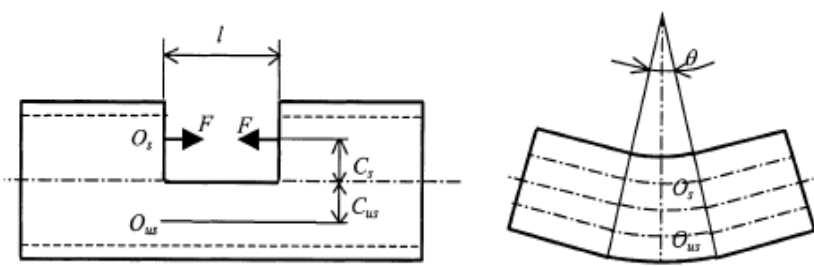

Figure 3: Simplified model for laser tube bending.
The heat energy generated by the laser for bending is:

$$
E=P \eta v t^{\prime}
$$

where $P$ is the laser power, $\eta$ is the absorption coefficient, $v$ is the ratio of the heating power to the input power and $t$ ' is the time period of the laser scan.

From the above, the laser tube bending angle is:

$$
\theta=(\pi l / 2 D)\left[(P \eta v \alpha / \omega D l t \rho c)-(\sqrt{ } 2-1) \sigma_{y} / E\right]
$$

where $\omega$ is the angular scanning speed, $\rho$ is the density of the material and $c$ is the specific heat of the material [40-13]. Imhan et al. are modify and enhance Hao and $\mathrm{Li}$ analytical by taken into account the effects of material specifications changes during laser tube bending due to the temperature rise and using Particle Swarm optimization to optimize the analytical and experimental results to reduce the mean absolute error [44].

\section{Numerical simulation of laser tube bending}

Simulation of the laser tube bending process is complex and difficult, as it involves numerous variables such as the heat flux of the laser beam, temperature distribution in the tube elements, changes in material properties and the influence of thermal strain on tube deformation. This was what Zhang and Liu [45] mentioned when they simulated the laser rectangular tube bending by developing a finite element model. Li and Yao [46] applied the nonlinear analysis in the model of the finite element analysis (FEA) due to the characteristics of the laser bending process of tubes such as cross-section ovalisation, wall thickness variation, bending radius and asymmetry. The numerical results were experimentally verified by utilizing the $\mathrm{CO}_{2}$ laser system with a maximum power of $1.5 \mathrm{~kW}$. Gaussian distribution

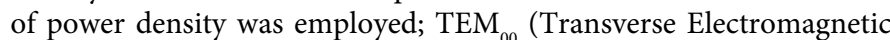
Mode). Majed et al. [47] proposed the FEM numerical simulation in ABAQUS. Three-dimensional heat-transfer elements and eight nodes DC3D8 were performed. The numerical results were validated by using the experimental results of the pulsed Nd-Yag laser. Jamil et al. [48] simulated the laser bending of the stainless steel tube and compared their results with those of published experimental data. The main underlying mechanism of laser forming was shown via simulations. BM was used to bend thin sheets of 0.5 and $0.9 \mathrm{~mm}$, while UM was used to bend thick plates measuring 1.5 and $2.0 \mathrm{~mm}$. Hsieh and Lin [39] solved the uncoupled thermal-mechanical problem in laser tube bending by employing a three-dimensional finite element method. Moreover, to analyze the thermal and mechanical behaviors in the laser bending of thin metal tubes via numerical simulation, several assumptions must be implemented, such as:

1. The material thermal properties are isotropic.

2. The distribution of laser intensity is in the Gaussian mode.

3. The heat losses due to free convection and thermal radiation are accounted for.

4. The heating phenomena due to phase changes are neglected.

5. The heat due to strain energy is neglected.

6. The Bauschinger's effect is neglected (which mean the material property where the material's stress/strain characteristics change as a result of microscopic).

7. The material is incompressible when plastically deformed.

8. The material is elastic-perfectly plastic. 
Furthermore, Li and Yao [49] developed a finite element model, which was experimentally validated. They examined the processing mechanism to understand the deformation characteristics such as the slightly protruded shape at the intrados, cross-section ovilization bending radius, wall thickness variation, and asymmetry. The $\mathrm{CO}_{2}$ laser machine was used to irradiate low carbon steel AISI 1010, emitting the maximum power of $1.5 \mathrm{~kW}$. Many of the process parameters were changed in this study to cover a wide range of variables; hence, the laser power was tested from 780 to $1500 \mathrm{~W}$, the angular scanning speed from 1.5 to $2.63 \mathrm{rad} / \mathrm{sec}$, the laser beam diameter between $11-15 \mathrm{~mm}$ and the maximum scanning angle from 180 to $322(\mathrm{deg})$.

\section{Parameters Affecting Laser Tube Bending}

Recent studies have focused on laser tube bending that can be affected by several parameters such as material, geometric and laser parameters [50]; they are either individually influential or mutually influential in the process direction.

\section{Material parameters' effect}

The material parameters can be classified into thermal parameters (such as thermal expansion coefficient, specific heat, thermal conductivity, etc.), physical parameters (such as density, absorption coefficient, etc.) and mechanical parameters (such as the yield stress, Young's modulus, etc.). The parameters are temperature dependent and have an obvious effect on the laser forming process. Hence, it is necessary to understand the effects of these parameters on laser bending angles and specify the optimal process parameters [35]. It can be concluded that the experimental and analytical approaches can help estimate the laser bending angle. Based on the previous discussion, the effect of material properties can be as follows:

- The thermal expansion coefficient is closely proportional to the bending angle.

- The increase of heat conductivity leads to the decrease of the bending angle.

- A larger bending angle can be obtained for the material with lower density and specific heat [51].

\section{Geometry parameters' effect}

Laser tube bending is distinguished by the complexity of its forming process due to geometrical conditions. Thus, the thickness and diameter of the tube guides the trend of the forming process and subsequently suggests an effective mechanism. Hence, the tube is irradiated by the buckling mechanism BM since the upper tube portion is thin and is regarded as a thin sheet. After the laser cut-off and cooling stage begins, the upper portion suffers shrinkage and shortening, which forces the lower portion of the tube to follow the difference in stress and bend the laser forward. Moreover, the tube diameter affects the speed of the laser process and the amount of energy penetrating the metal due to the change of interaction area. Hao proposed a formula for the bending angle based on plastic deformation, which was experimentally validated [52]. It can be seen that the bending angle is the result of tube dimension, material properties, laser scanning speed and laser power. Wang and others utilized a strategy involves a method for calculating the geometric curvature of the curved tubes and investigate both plane bending and three-dimension bending as well as the results verified experimentally [53].

\section{Laser parameters' effect}

Laser parameters are the most important factors in the laser tube bending process due to being flexible, while its properties are controllable. The literature had pointed out that the effect of the laser power is linearly proportional to the value of the angle produced, while the scanning speed is inversely proportional. To be more specific, one must analyze the laser beam and scanning characteristics. Thus, the laser beam geometry can be modified to vary the temperature distribution without changing the input power or the scanning speed. The longest beam dimensions in the scanning direction realize higher temperatures, although the fact is that the power intensity and beam effective area are similar for all beams. This is accomplished by the longer beam-material interaction for a beam with longer dimension in the scanning direction. It is also important to note that the narrower the beam's transverse width, the higher the cooling rate after the beam leaves the location point as shown in Figure 4 [25].

The general analytical expressions for laser tube bending are mostly considered the circumferential scanning schemes [39,40,43,46,49,52], assuming a two-dimensional temperature distribution and temperature independent material properties. The technique of axial scanning was illustrated in Figure 5 where the laser beam defocused on the upper surface of the tube and the CNC table was moved [54]. In addition, Zhang et al. [55] proposed a numerical simulation for tube bending with four lasers scanning schemes involving pointsource circumferential scanning, pulsed line-source axial procession, and line-source axial scanning; with and without water cooling. It is clear that scanning schemes are significantly influential vis-à-vis laser tubes bending. Comparing the axial scanning scheme results in greater angular deformation when compared to the circumferential scanning scheme, the bending angle produced is greater, which would decrease the number of scans required [54].

The scanning path is a crucial factor in laser tube bending for obtaining 3D shapes and 2D curvature shapes. Wang et al. [53] created model calculations and experimentally verified the bending of straight tubes into a $2 \mathrm{D}$ sinusoidal and a $3 \mathrm{D}$ helical/coil-shaped tube. It was shown that the model's calculations were in excellent agreement

\begin{tabular}{|c|c|c|c|c|c|c|}
\hline CASE & A & B & $\mathrm{C}$ & D & E & CIRCULAR \\
\hline \multirow{2}{*}{$\begin{array}{l}\text { Beam } \\
\text { profile }\end{array}$} & & & & & & \\
\hline & $\begin{array}{l}\text { Width }=25 \mathrm{~mm} \\
\text { Length }=8 \mathrm{mmm}\end{array}$ & $\begin{array}{l}\text { Width }=20 \mathrm{~mm} \\
\text { Length }=10 \mathrm{~mm}\end{array}$ & $\begin{array}{l}\text { Width=14.14mm } \\
\text { Length }=14.14 \mathrm{~mm}\end{array}$ & $\begin{array}{l}\text { Width }=10 \mathrm{~mm} \\
\text { Length }=20 \mathrm{~mm}\end{array}$ & $\begin{array}{l}\text { Width }=8 \mathrm{~mm} \\
\text { Length }=25 \mathrm{~mm}\end{array}$ & Diameter $=16 \mathrm{~mm}$ \\
\hline Aspect ratio & 3.125 & 2.0 & 1.0 & 0.5 & 0.32 & - \\
\hline
\end{tabular}

Figure 4: Beam geometries with similar effective areas. 


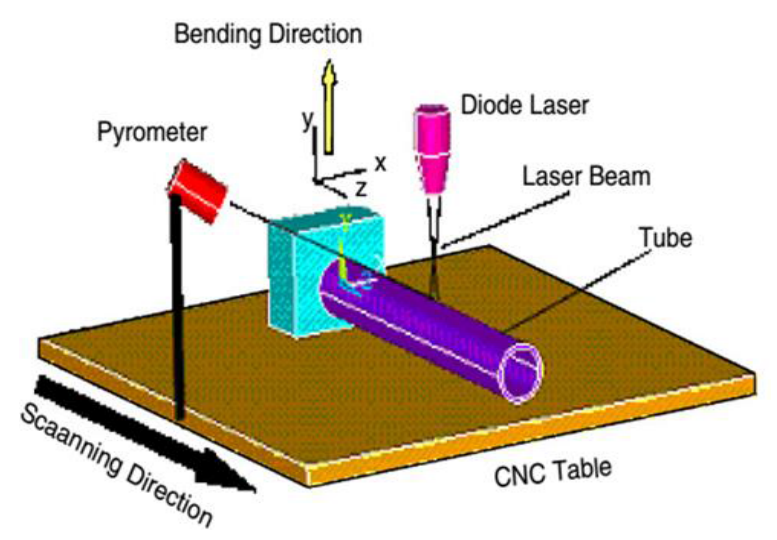

Figure 5: The axial scanning scheme.

(a)

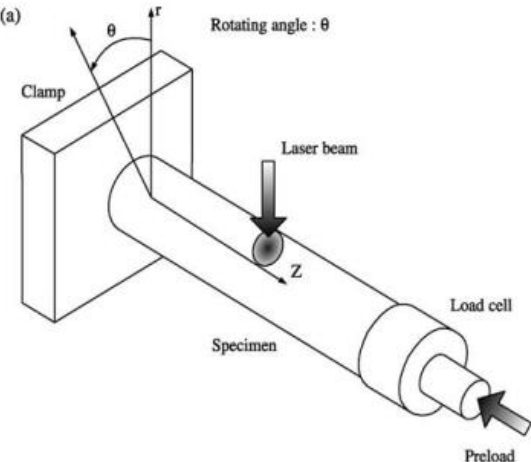

(b)

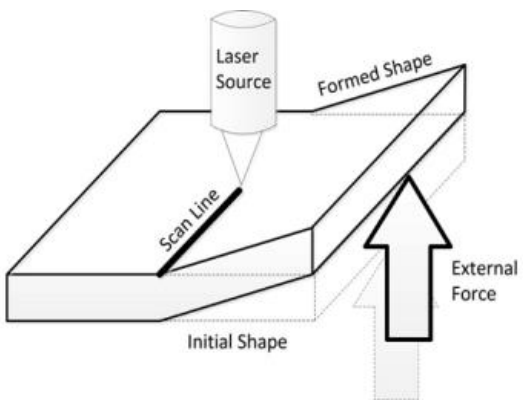

Figure 6: External force assisting laser forming: (a) Laser tube bending and (b) Laser sheet forming.

with the experimental data. The results provide a theoretical and technological basis, with a wide application for the laser bending of steel tubes. Moreover, the laser beam diameter is another significant factor in laser tube bending. Lawrence et al. [11] pointed out that the laser beam diameter is one of the parameters that must be taken into account in the process design to avoid the melting temperature of the material surface and its effect on the selected laser processing mechanism (Figure 6).

\section{Size and edge effects}

Numerous researchers had studied the size and edge effects on the sheet material. This can be reflected on tube edge effects such as diameter, thickness, and length of the specimen. The size effect is important for quality control of products produced by laser forming. Cheng et al. [56] investigated the size effect on the laser forming of lowcarbon steel sheet to better comprehend the deformation characteristics and provide a useful tool to predict the bending deformation. The result was that the bending angle decreases when the sheet width increases, while the sheet length remains constant. And the bending angle increases when the sheet length increases, while the sheet width remains constant. Kyrsanidi et al. [57] calculated the distortion angle with clear dependency on the width to some extent; it then becomes width independent when the width exceeds $400 \mathrm{~mm}$. Therefore, a new mathematical model was duly developed. Jha et al. [58] studied the edge effect on the laser bending of AISI 304 stainless steel using the $\mathrm{CO}_{2}$ laser with maximum power of $10 \mathrm{~kW}$. The study demonstrated that the edge effect totally depends on process parameters, such as the average laser power and scanning speed. It was discovered that laser bending can sometimes render a multi-curvature on a single laser scan path and laser scanning speed.

\section{Cooling effect}

The difference in temperatures and their distribution on the specimen surface or thickness leads to an understanding of stress flow and strain deformation. Hence, cooling in either the single or multiscanning laser forming can increase the temperature difference and decrease the process time in multi-scan formation. Cheng and Yao [59] experimentally and numerically examined the effect of cooling on single-scan and multi-scan laser forming processes. The cooling effects under various conditions (such as different laser powers, scanning speed, nozzle offset and cooling air pressure) were duly investigated. The cooling effects were examined on the changing microstructure and other mechanical properties. The focus was to investigate the cooling effect on the deformation mechanism, including the competing effects on temperature and flow stress. The investigation illustrated that forced cooling with multi-scan laser forming reduces process time and prevents unwanted effects on microstructural changes and mechanical properties.

\section{Others Factors}

\section{Large tubes bending}

The mechanical method is used to bend tubes with a thin wall but is incapable of bending tubes with diameters larger than $50 \mathrm{~mm}$ due to difficulties in producing templates, as well as, the associated high costs. Hence, the extrados portion stretches while the intrados portion 
Citation: Imhan KI, Baharudin BTHT, Zakaria A, Ismail MISB, Alsabti NMH, et al. (2018) Features of Laser Tube Bending processing based on Laser Forming: A Review. J Laser Opt Photonics 5: 174. doi: 10.4172/2469-410X.1000174

suffers from wrinkling rather than the distortion of the cross section [2]. Zhang et al. [60] suggested the laser light for bending large tube diameters that are 6 inches with a thin wall (referred to as the wall factor $>80$ ), which can be defined as the ratio between outer diameter OD and wall thickness. The challenges were solved using plugs to avoid ovalisation, active cooling to increase the process speed, surface coating to enhance the absorptivity and a rectangular laser beam to produce a uniform thermal profile. Tube diameters over $300 \mathrm{~mm}$ were bent 5 degrees after 200 scans for 20 minutes at $200 \mathrm{~mm}$ in length.

\section{Preload and laser assistance}

The laser assisted forming techniques, such as deep drawing or hydroforming of the preload force, help laser tube bending to increase bulging. Hsieh and Lin [61] simulated and compared the transient state of a thin metal tube under buckling with axial preloads due to a laser beam with measurements such as the surface temperature and axial force taken during the experiment. The problem was assumed to be a 3D uncoupled thermo-elasto plastic. The study showed that using a varying preload from 100-400 $\mathrm{N}$ can increase the bending angle for the same laser power and scanning speed. Moreover, during the forming processes, the deformation rates increased and quite easily caused cracks and collapse; especially for brittle materials such as titanium or magnesium, which makes forming difficult [62]. Hence, the laser is used to heat the localized area that is to be formed to change the material specifications and assist deep drawing or bending. Roohi et al. [63] accompanied the laser beam with an external mechanical force to gain a 90-degree bending angle from bending a plate of the Al-5005 alloy by assisting this external force to the laser forming process.

\section{Conclusion}

This review clarified the mechanisms employed in laser forming in general, and laser tube bending in particular. Methods of the investigation were reviewed, and analytical models controlling the process were then described. The principle of laser tube bending was explained, and the effects of different parameters (such as material, geometry, laser, edge and cooling effects) were then discussed. Furthermore, large tube bending and preload, or laser assistance, were presented as well. The summary of conclusions is as the following:

1. The TGM is used to bend thick sheets with small laser beam diameter.

2. The BM is used to bend the thin sheets and tubes with large laser beam diameter comparison to the thickness.

3. The bending angle is relatively small, and multi-scans should be applied to obtain the desired angle.

4. Laser parameters such as laser power, laser scanning speed as well as laser beam diameter are significantly affecting the laser tube bending process.

5. Laser tube bending can bend large tube diameter more than $300 \mathrm{~mm}$.

6. Preload is used to bend both plates and tubes; the bending angle achieved was 90 degrees.

7. Please be informed that the authors declare they have no conflict of interest.

\section{References}

1. Kannatey-Asibu E (2009) Principles of laser materials processing. John Wiley \& Sons Vol: 4.
2. He Y, Li H, ZHANG Z, Guangjun LI (2012) Advances and trends on tube bending forming technologies. Chinese Journal of Aeronautics 25: 1-12.

3. Vollertsen F, Sprenger A, Kraus J, Arnet H (1999) Extrusion, channel, and profile bending: a review. Journal of Materials Processing Technology 87: 1-27.

4. Gollo MH, Mahdavian S, Naeini HM (2011) Statistical analysis of parameter effects on bending angle in laser forming process by pulsed Nd: YAG laser. Optics \& Laser Tech 43: 475-482

5. Shi Y, Shen H, Yao Z, Hu J (2007) Temperature gradient mechanism in laser forming of thin plates. Optics \& Laser Tech 39: 858-863.

6. Smith T et al. (2012) Finite element model of pulsed laser forming. 13th International Symposium on Laser Precision Micro fabrication.

7. Akinlabi ET, Shukla M, Akinlabi SA (2012) Laser Forming of Titanium and Its Alloys-An Overview. Proceedings of World Academy of Science, Engineering and Technology. World Academy of Science, Engineering and Technology (WASET).

8. Shi Y, Shen H, Yao Z, Hu J (2006) Numerical investigation of straight-line laser forming under the temperature gradient mechanism. Acta Metallurgica Sinica (English Letters). 19: 144-150.

9. Magee J, Watkins K, Edwardson P, Dearden G, French P (2001) Laser forming of aerospace alloys. AMTC, Seattle, Society of Automotive Engineers, Paper, (2001-01) pp: 2610.

10. Carlone P, Palazzo GS, Pasquino R (2008) Inverse analysis of the laser forming process by computational modelling and methods. Computers \& Mathematics with Applications 55: 2018-2032.

11. Lawrence JR (2010) Advances in laser materials processing: Technology, research and application. Elsevier.

12. Dearden G, Edwardson S (2003) Laser assisted forming for ship building. SAIL, Williamsburg, VA, pp: 2-4.

13. Akinlabi SA, Akinlabi ET (2013) Experimental Investigation of Laser beam forming of Titanium and Statistical Analysis of the Effects of Parameters on Curvature. IMECS Vol: 2.

14. Mucha Z, Hoffman A, Mucha S, Kalita W (1997) Laser forming of thick free plates. Laser Assisted Net Shape Engineering, Proceedings of the LANE 97: 383-392.

15. Jamil MC, Sheikh M, Li L (2011) A study of the effect of laser beam geometries on laser bending of sheet metal by buckling mechanism. Optics \& Laser Technology 43: 183-193.

16. Hu Z, Kovacevic R, Labudovic M (2002) Experimental and numerical modeling of buckling instability of laser sheet forming. Inter J Machine Tools Manufacture 42: $1427-1439$.

17. Li W, Yao YL (2000) Buckling based laser forming process: concave or convex ICALEO 2000: Laser Materials Processing Conference.

18. Geiger M, Merklein M, Pitz M (2004) Laser and forming technology-an idea and the way of implementation. J Mate Process Tech 151: 3-11.

19. Steen W, Mazumder J (2010) Laser material processing: Springer Science \& Business Media, springer.

20. Dowden J (2009) The Theory of Laser Materials Processing: Heat and Mass Transfer in Modern Technology. Springer Science \& Business Media Vol: 119

21. Li L, Yanbin C, Xuyou W, Shangyang L (2009) FEM simulation for laser forming processing. Acta Metallurgica Sinica (English Letters) 17: 317-322.

22. Hennige T, Holzer S, Vollertsen F, Geiger M (1997) On the working accuracy of laser bending. Journal of Materials Processing Technology 71: 422-432

23. Lawrence J, Schmidt MJ, Li L (2001) The forming of mild steel plates with a $2.5 \mathrm{~kW}$ high power diode laser. International Journal of Machine Tools and Manufacture 41: 967-977.

24. Gollo MH, Naeini HM, Arab NM (2011) Experimental and numerical investigation on laser bending process. JCARME 1: 45-52.

25. Liu S, Fang X, X-r FAN (2004) Experiment investigation on rules of laser tube bending. Laser Technology 4: 001.

26. Griffiths JD (2012) Modelling of laser forming at macro and micro scales University of Liverpool Liverpool.

27. Shen H, Vollertsen F (2009) Modelling of laser forming-An review. 
Citation: Imhan KI, Baharudin BTHT, Zakaria A, Ismail MISB, Alsabti NMH, et al. (2018) Features of Laser Tube Bending processing based on Laser Forming: A Review. J Laser Opt Photonics 5: 174. doi: 10.4172/2469-410X.1000174

Computational Materials Science 46: 834-840.

28. Yau C, Chan K, Lee W (1997) A new analytical model for laser bending. Laser assisted net shape engineering.. Meisenbach Bamberg.

29. Cheng $P$, Lin $S$ (2001) An analytical model to estimate angle formed by laser. Journal of Materials Processing Technology 108: 314-319.

30. Cheng P, Fan Y, Zhang J, Lawrence Yao Y, Mika David P (2006) Laser forming of varying thickness plate-Part I: Process analysis. Journal of Manufacturing Science and Engineering 128: 634-641.

31. Li W, Yao YL (2000) Numerical and experimental study of strain rate effects in laser forming. Journal of Manufacturing Science and Engineering 122: 445451.

32. Kyrsanidi AK, Kermanidis TB, Pantelakis SG (1999) Numerical and experimental investigation of the laser forming process. Journal of Materials Processing Technology 87: 281-290.

33. Lok MA, Hideki A (2013) An analytical model for calculating the temperature distribution and determining the laser forming mechanism. ICOMM.

34. WANG Xy, Wei-xing XU, Wen-ji XU, Ya-fengHU, Yan-de LIANG, et al. (2011) Simulation and prediction in laser bending of silicon sheet. Transactions of Nonferrous Metals Society of China. 21: 188-s193.

35. Safari M (2014) Numerical Investigation of the Effect of Process and Sheet Parameters on Bending Angle in the Laser Bending Process. World Journa of Mechanics.

36. Moslemi Naeini $H$, Mazdak HLVP S, Mousavi Hondori H (2007) TGM Laser Forming Process for Sheet Metals Using Experimental and FEM Analysis Approach. 4th International Conference and Exhibition on Design and Production of MACHINES and DIES/MOLDS.37. Chen G, Xu X (2001) Experimental and 3D finite element studies of CW laser forming of thin stainless steel sheets. Journal of Manufacturing Science and Engineering 123: 66-73.

37. Jamil MC, Imam Fauzi ER, Juinn CS, Sheikh MA (2015) Laser bending of prestressed thin-walled nickel micro-tubes. Optics \& Laser Technology 73: 105117

38. Hsieh HS, Lin J (2005) Study of the buckling mechanism in laser tube forming. Optics \& Laser Technology 37: 402-409.

39. N Hao, Li L (2003) An analytical model for laser tube bending. Applied surface science 208: 432-436.

40. Duley W (2012) CO2 lasers effects and applications: Elsevier.

41. Guan Y, Yuan G, Sun S, Zhao G, (2013) Process simulation and optimization of laser tube bending. The International Journal of Advanced Manufacturing Technology 1-10.

42. Hao N, Li L (2003) Finite element analysis of laser tube bending process. Applied Surface Science 208: 437-441.

43. Imhan KI, Hang Tuah Baharudin BT, Zakaria A, Ahmad AK (2017) Investigation of material specifications changes during laser tube bending and its influence on the modification and optimization of analytical modeling. Optics \& Laser Technology 95: 151-156.

44. Zhang $P$, Liu $H$ (2010) Thermal deformation behaviors of laser rectangular tube bending. (CMCE) Inter Conference IEEE.

45. Li W, Yao YL (2001) Laser bending of tubes: mechanism, analysis, and prediction. Journal of Manufacturing Science and Engineering 123: 674-681.
46. Majed A, Ahmadi F, Farzin M (2009) Experiment and finite element simulation of laser bending of tubes. Proceedings of the Iran Conference Manufacturing Engineering, Birjand, Iran.

47. Jamil MC, Sheikh M, Li L (2012) A Finite Element Study of Buckling and Upsetting Mechanisms in Laser Forming of Plates and Tubes. Dynamic Methods and Process Advancements in Mechanical, Manufacturing, and Materials Engineering pp: 140.

48. Li W, Yao Y (2000) Numerical and experimental investigation of laser induced tube bending. ICALEO 2000: Laser Materials Processing Conference.

49. Geiger M (1994) Synergy of laser material processing and metal forming. CIRP Annals-Manufacturing Technology 43: 563-570.

50. Guan Y, Sun SY, Zhao G, Luan Y (2005) Influence of material properties on the laser-forming process of sheet metals. Journal of Materials Processing Technology 167: 124-131.

51. Hao N (2010) On the process parameter of laser tube bending. In Mechanic Automation and Control Engineering (MACE), 2010 International Conference on IEEE.

52. Wang X, Luo YH, Wang J, Xu WJ, Guo DM (2014) Scanning path planning for laser bending of straight tube into curve tube. Optics \& Laser Technology 56: 43-51.

53. Safdar S, Li L, Sheikh MA, Liu Z (2007) Finite element simulation of laser tube bending: Effect of scanning schemes on bending angle, distortions and stress distribution. Optics \& Laser Tech 39: 1101-1110.

54. Zhang J, Cheng P, Zhang W, Graham M, Jones J, et al. (2006) Effects of scanning schemes on laser tube bending. Journal of Manufacturing Science and Engineering 128: 20-33.

55. Cheng $P$, Lawrence $Y$, Yao, Liu C, Pratt D, Fan $Y$ (2005) Analysis and prediction of size effect on laser forming of sheet metal. Journal of Manufacturing Processes 7: 28-41.

56. Kyrsanidi AK, Kermanidis TB, Pantelakis SG (2000) An analytical model for the prediction of distortions caused by the laser forming process. Journal of Materials Processing Technology 104: 94-102.

57. Jha GC, Nath A, Roy S (2008) Study of edge effect and multi-curvature in laser bending of AISI 304 stainless steel. Journal of Materials Processing Technology 197: 434-438.

58. Cheng J, Yao YL (2001) Cooling effects in multiscan laser forming. J Manufacturing Processes 3: 60-72.

59. Zhang W, Jones M, Graham M, Farrell B, Azer M, et al. (2005) Large diameter and thin wall laser tube bending. 24th International Congress on Applications of Lasers and Electro-Optics, ICALEO.

60. Hsieh HS, Lin J (2005) Study of the buckling mechanism in laser tube forming with axial preloads. International Journal of Machine Tools and Manufacture 45: $1368-1374$.

61. Kratky A (2006) Laser assisted forming techniques. XVI International Symposium on Gas Flow, Chemical Lasers, and High-Power Lasers. Inter Society for Optics and Photonics.

62. Roohi AH, Gollo MH, Naeini HM (2012) External force-assisted laser forming process for gaining high bending angles. Journal of Manufacturing Processes 14: 269-276. 